\title{
Abnormal Brain Connectivity in Carpal Tunnel Syndrome Assessed by Graph Theory
}

This article was published in the following Dove Press journal:

Journal of Pain Research

\author{
Xiang-Xin Xing ${ }^{1,2, *}$ \\ Xu-Yun Hua ${ }^{3,4, *}$ \\ Mou-Xiong Zheng ${ }^{3, *}$ \\ Jia-Jia $\mathrm{Wu}^{2}$ \\ Bei-Bei Huo ${ }^{1,2}$ \\ Jie $M a^{1,2}$ \\ Zhen-Zhen Ma ${ }^{1,2}$ \\ $\mathrm{Si}-\mathrm{Si} \mathrm{Li}^{1,2}$ \\ Jian-Guang $X u^{\prime}$
}

'School of Rehabilitation Science, Shanghai University of Traditional Chinese Medicine, Shanghai, People's Republic of China; ${ }^{2}$ Department of Rehabilitation Medicine, Yueyang Hospital, Shanghai University of Traditional Chinese Medicine, Shanghai, People's Republic of China; ${ }^{3}$ Department of Traumatology and Orthopedics, Yueyang Hospital, Shanghai University of Traditional Chinese Medicine, Shanghai, People's Republic of China; ${ }^{4}$ Yangzhi Rehabilitation Hospital, Tongji University, Shanghai, People's Republic of China

*These authors contributed equally to this work

\begin{abstract}
Introduction: Numerous resting-state functional magnetic resonance imaging (fMRI) researches have indicated that large-scale functional and structural remodeling occurs in the whole brain despite an intact sensorimotor network after carpal tunnel syndrome (CTS). Investigators aimed to explore alterations of the global and nodal properties that occur in the whole brain network of patients with CTS based on topographic theory.

Methods: Standard-compliant fMRI data were collected from 27 patients with CTS in bilateral hands and 19 healthy control subjects in this cross-sectional study. The statistics based on brain networks were calculated the differences between the patients and the healthy. Several topological properties were computed, such as the small-worldness, nodal clustering coefficient, characteristic path length, and degree centrality.

Results: Compared to those of the healthy controls, the global properties of the CTS group exhibited a decreased characteristic path length. Changes in the local-level properties included a decreased nodal clustering coefficient in 6 separate brain regions and significantly different degree centrality in several brain regions that were related to sensorimotor function and pain.

Discussion: The study suggested that CTS reinforces global connections and makes their networks more random. The changed nodal properties were affiliated with basal gangliathalamo-cortical circuits and the pain matrix. These results provided new insights for improving our understanding of abnormal topological theory in relation to the functional brain networks of CTS patients.

Perspective: This article presents that the CTS patients' brain with a higher global efficiency. And the significant alterations in several brain regions which are more related to pain and motor processes. The results provided effective complements to the neural mechanisms underlying CTS.
\end{abstract}

Keywords: small-worldness, nodal clustering coefficient, degree centrality, pain, circuits

\section{Introduction}

Carpal tunnel syndrome (CTS), a usual entrapment neuropathy, results from increased compression of the median nerve within the carpal tunnel. According to the reported prevalence of quality standards subcommittee of the American Academy of Neurology, $10 \%$ of people in the US will develop CTS at some point. ${ }^{1}$ In the early stage, CTS is characterized as intermittent, nocturnal paresthesias and dysaesthesias, particularly pain, which develops into a loss of sensation and muscle atrophy. ${ }^{2}$ This syndrome usually occurs in the hands but can spread to the shoulder in severe cases. ${ }^{3}$ Clinical evidence has suggested that occupation, arthritis and some hormonal pathophysiological changes are the risk factors for CTS. Interestingly, these hormonal alterations may explain why women are at higher risk of CTS than men. ${ }^{4}$
Correspondence: Jian-Guang Xu School of Rehabilitation Science, Shanghai University of Traditional Chinese Medicine, No. 1200 Cailun Road, Shanghai, People's Republic of China Tel +86-2I-5I32209|

$\mathrm{Fax}+86-2 \mathrm{I}-5 \mathrm{I} 322042$

Email xjg@shutcm.edu.cn
Journal of Pain Research 2021:14 693-701 
However, previous researchers found that the clinical symptoms of CTS are related not only to peripheral nerve function but also to central nervous plasticity. ${ }^{5,6}$ Investigators have revealed that CTS patients experience widespread central sensitization. ${ }^{7}$ Several resting-state functional magnetic resonance imaging (rs-fMRI) researches have confirmed that significant differences in the function and structure of the brain exist for CTS relative to healthy groups. ${ }^{8,9}$ For example, the cortical representations of digits in the primary sensory cortex (S1) were significantly amplified, reduced, or shifted in CTS patients. ${ }^{10}$ The gray matter volume in the primary sensory cortex, thalamic pulvinar and frontal poles was reduced. ${ }^{6}$ Although brain remodeling after peripheral nerve injury (PNI) has recently attracted more attention, few researchers have focused on alterations at the wholebrain-network level. ${ }^{11}$

Structurally, the associated brain regions were distant and scattered, but studies have suggested that the complicated anatomical and functional networks are made up of neurons found across the entire brain. ${ }^{12}$ With the development of neuroimaging research, relevant fMRI studies have proposed a theory about network organizational and topological properties in the brain ${ }^{13}$ and constructed a theoretical framework to evaluate complex and robust brain networks. ${ }^{14}$ The theory, graph theoretical analysis, has been widely adopted to illuminate brain complexity and topology, and it is considered an effective methodological approach with which to quantify the structural and functional central neural systems. Accumulated evidence has suggested that alterations of different topological properties can be caused by various neuropsychiatric disorders. ${ }^{15}$ Therefore, in the present study, we aimed to investigate the topological properties in CTS patients by using rs-fMRI with graph theoretical analysis.

The main hypothesis as follows: (1) all the participants, including CTS patients and healthy controls (HCs), would present small-world properties; (2) compared to HCs, the CTS patients would display disordered topological properties in the nodal and functional networks of the brain; (3) the disrupted neural circuits of CTS patients would be explained by topological properties. To confirm these above hypotheses, rs-fMRI data were acquired from 27 CTS and 18 HCs. We used graph theory to systematically construct and analyze the brain functional networks and nodal properties.

\section{Methods}

\section{Participants and Clinical Assessments}

Initially, fifty-three right-handed participants, including 30 patients with CTS and 23 HCs subjects, were recruited from the Yueyang Hospital of Integrated Traditional Chinese and Western Medicine, Shanghai University of Traditional Chinese Medicine. Written informed consent was obtained from each participant. The current study was approved by the Medical Ethics Committee of Yueyang Hospital. All work was performed in accordance with the Helsinki Declaration. However, seven participants were excluded in the next steps.

The patients with CTS presented at a stage with specific neurological signs and delayed motor/sensory conduction velocity. The inclusion criteria were as follows: (1) paresthesia and/or numbness in the territories which was innervated by median nerve, weakness in the wrist and/or finger, pain at night, and/or thenar atrophy in bilateral hands for more than 3 months according to the rules published by American College of Occupational and Environmental Medicine, 2011; (2) reverse Phalen's and positive Phalen's signs; (3) the media nerve motor latency above $3.7 \mathrm{~ms}$. The exclusion criteria of all participants were as follows: (1) the history of peripheral neuropathies or cerebral diseases, and (2) MRI contraindications. All the patients underwent an assessment by electromyography to evaluate the motor latency of the median nerve. A longer latency denoted more severe disability of the affected hand. Meanwhile, The patients completed the Boston Carpal Tunnel Syndrome Questionnaire (BCTQ). To ensure the these criteria, one professional hand surgeon was involved throughout the diagnosis of all patients and HCs.

\section{fMRI Data Acquisition and Preprocessing}

Before scanning, the participants were told to remain at rest and awake without thinking or falling asleep. Their heads were fixed to reduce any head motion. The images were collected by using a Magnetom Trio A 3T MR Scanner (Siemens AG, Erlangen, Germany). Rs-fMRI images were acquired using a Gradient Echo-Echo Planar Imaging (GRE-EPI) sequence with the following parameters: interleaved scanning order; Field of view $=240 \times 240 \mathrm{~mm}^{2}$; matrix size $=64 \times 64$; slice number $=43$; Time of repetition $/$ echo $=3000 / 30 \mathrm{~ms}$; flip angle $=90^{\circ}$; slice thickness $=3.0 \mathrm{~mm}$; acquisition voxel size $=3.4 \times 3.4 \times 3.2 \mathrm{~mm}^{3}$; and number of repetitions $=240$. The Graph-theoretical Network Analysis Toolkit (GRETNA, 
www.nitrc.org) was applied for data preprocessing as follows: remove the first 10 volume, slice timing, realignment, normalization by the EPI template of the Montreal Neurological Institute $(\mathrm{MNI})$ space, resampling to $3.0 \times 3.0 \times 3.0 \mathrm{~mm}^{3}$, spatial smoothing with a 4-mm full-width at half-maximum Gaussian kernel, temporal detrending and filtering (0.01-0.1 $\mathrm{Hz}$ ), regressing out of covariates. Finally, the data from four HCs and three patients were excluded because of excessive head motion (more than $2^{\circ}$ and $2 \mathrm{~mm}$ ) or serious artifacts. The data of forty-six participants were analyzed in the next steps.

\section{Brain Network Construction and Analysis}

And next, the GRETNA was used to construct the functional brain network. According to the Anatomical Automatic Labeling (AAL), which supported by MNI, parcellated the whole cerebral cortex into 90 cortical and subcortical anatomically defined regions. Additionally, the mean time series of each region was extracted. And then, the pearson's correlation coefficients of each pair of regions in AAL were computed, and Fisher's $z$ transformation was used to convert the data to $z$-values which were near to normally distributed. A binary connection matrix, converted by the $z$-values with a selected threshold of the relation matrix, was used to construct the graphic model of the functional network. According to previous study, the sparsity was set at $10-46 \%$, with an interval of $0.01 .{ }^{11} \mathrm{We}$ also used the GRETNA to calculate the topological properties in the network, and the global properties of the functional network were represented by the following measures: smallworldness, clustering coefficient $(C p)$ and characteristic path length $(L p)$. And we used the nodal clustering coefficient and degree centrality (DC) to demonstrate the nodal properties in the functional network.

\section{Statistical Analysis}

The two-sample $t$-tests based on Statistical Package for Social Sciences (SPSS 21.0) software was used to assess the difference between the CTS and the HCs in demographic characteristics. The significance level of the global properties in the brain network between the CTS and the HC group was set at $p<0.05$, uncorrected. The test procedure was performed at each selected threshold sparsity $(0.1-0.46)$ to detect significant differences between the two sets. The DC values of the patients group were extracted. And then, the Pearson's correlation analysis was performed between the DC values and the BCTQ.

\section{Results}

\section{Demographic Data}

The demographic data of the remaining participants are shown in Table 1 . There were no statistically significant differences in age or gender ratio between the two groups $(p>0.05)$.

\section{Small-Worldness}

Figure 1 shows the comparison of the global measures between the CTS patients and the HCs. Both the CTS group and the HCs demonstrated small-world organization in the functional network, which was characterized by normalized $\mathrm{Cp}(\gamma)>1$ and normalized $\operatorname{Lp}(\lambda) \approx 1$. However, the CTS

Table I Demographic Information and the Clinical Assessments of the Subjects

\begin{tabular}{|c|c|c|c|c|}
\hline & \multicolumn{2}{|c|}{ CTS Patients } & HCs & $P$ value \\
\hline Age & \multicolumn{2}{|c|}{$53.56 \pm 8.50$} & $56.89 \pm 6.98$ & 0.166 \\
\hline Gender(M/F) & \multicolumn{2}{|c|}{$2 / 25$} & $3 / 16$ & 0.676 \\
\hline BCTQ score (Symptom) & \multicolumn{2}{|c|}{$27.51 \pm 5.62$} & - & - \\
\hline BCTQ score (Daily life) & \multicolumn{2}{|c|}{$17.37 \pm 4.50$} & - & - \\
\hline Median nerve motor latency(ms) & $\begin{array}{c}\text { Right } \\
5.04 \pm I .2 I\end{array}$ & $\begin{array}{c}\text { Left } \\
5.48 \pm 2.04\end{array}$ & - & - \\
\hline Median nerve sensory latency $(\mathrm{ms})$ & $\begin{array}{c}\text { Right } \\
3.23 \pm I .50\end{array}$ & $\begin{array}{c}\text { Left } \\
3.23 \pm 0.86\end{array}$ & - & - \\
\hline M_motor_NCV $(\mathrm{m} / \mathrm{s})$ & $\begin{array}{c}\text { Right } \\
37.00 \pm 9.12\end{array}$ & $\begin{array}{c}\text { Left } \\
35.44 \pm 10.56\end{array}$ & - & - \\
\hline M_sensory_NCV(m/s) & $\begin{array}{c}\text { Right } \\
4|.2| \pm 7.53\end{array}$ & $\begin{array}{c}\text { Left } \\
39.39 \pm 8.19\end{array}$ & - & - \\
\hline
\end{tabular}

Abbreviations: $M$, male; F, female. 


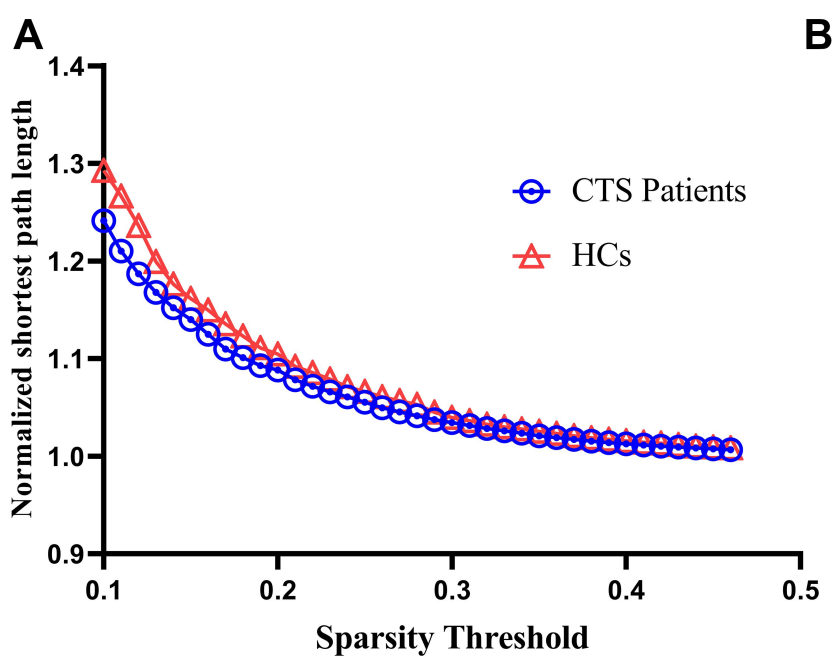

B
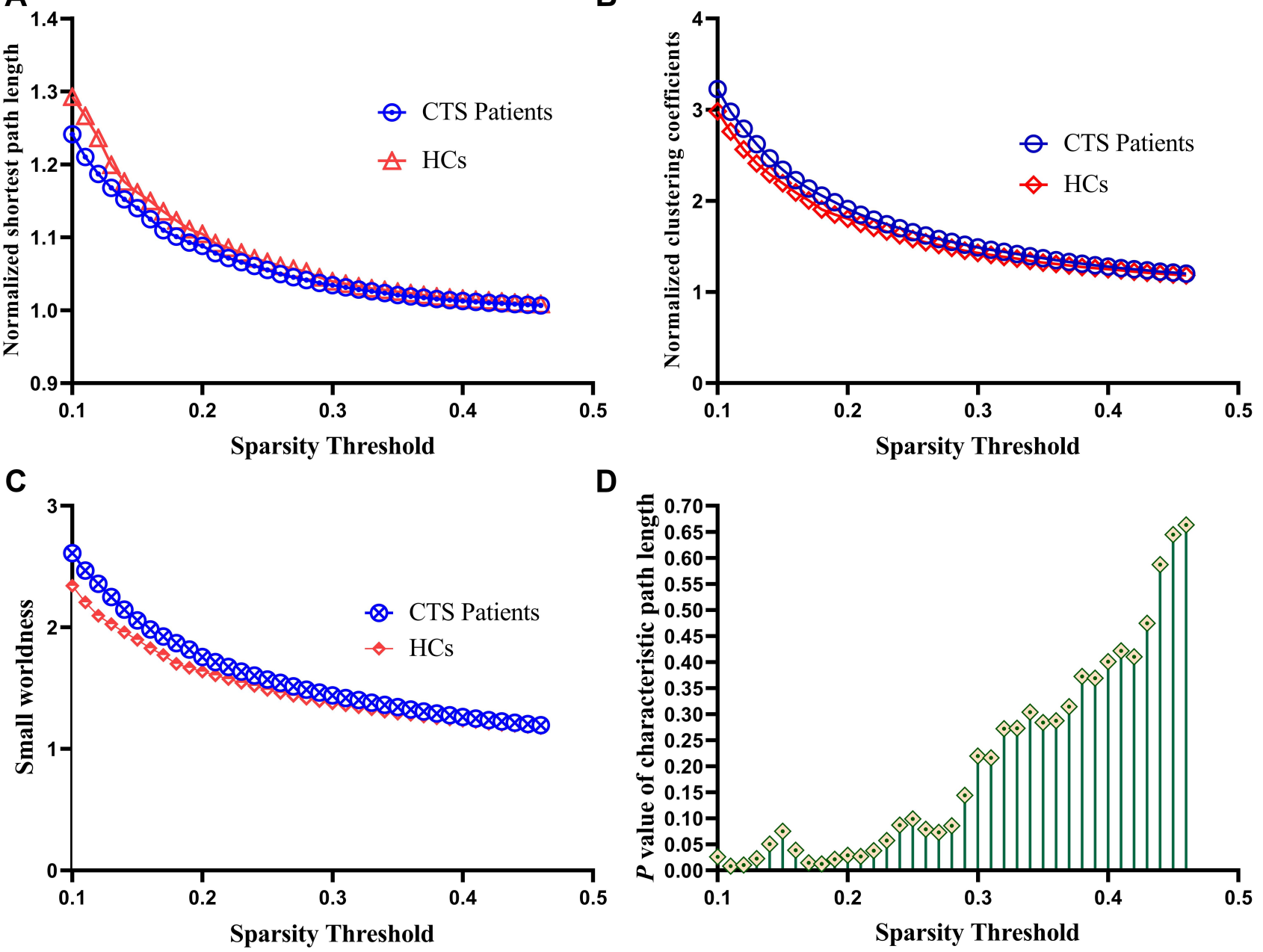

Figure I The small-world parameters of the global cerebral resting-state functional network between the CTS patients and HCs at a sparsity range of $0.1-0.46$. (A) Normalized characteristic path length $(\lambda)$, compared with that of the HCs, the $\lambda$ of the CTS was significantly decreased $(P<0.05)$. (B) Normalized clustering coefficients $(\gamma)$, compared with that of the HCs, the $\gamma$ of the CTS was not significantly difference $(P>0.05)$. (C) Small-worldness $(\sigma)$, compared with that of the HCs, the $\sigma$ of the $C T S$ was significantly increased $(P<0.05)$. (D) The $\mathrm{P}$ value of characteristic path length: the CTS patients had a significantly lower LP than the HCs over a wide range of sparsity thresholds $(P<0.05)$.

Abbreviations: $\mathrm{HCs}$, healthy controls; CTS, carpal tunnel syndrome.

patients had a significantly lower Lp than the HCs over a wide range (0.1 0.22) of sparsity thresholds ( $p<0.05$, uncorrected), but the $\mathrm{Cp}$ and $\gamma$ showed no significant alteration. After solving the equation small-worldness $(\sigma)=\gamma / \lambda$, increased $\sigma$ was also identified in the CTS patients over a wide range of thresholds too.

\section{Nodal Characteristics in the Functional Networks}

Compared to the HCs, the significantly decreased nodal shortest paths in 6 discrete regions were presented in the CTS group ( $p<0.05$; Figure 2A, Table 2). The degree centrality (Figure 2B, Table 3 ) was increased in the right middle orbital frontal and parahippocampal, caudate, bilateral amygdala and decreased in the right cuneus, right middle occipital, right thalamus, bilateral opercularis rolandic, and bilateral supplementary motor area, left olfactory.

\section{Correlations Between the DC and BCTQ}

Figure 3 shows the correlation between $\mathrm{DC}$ and the functional status scales of $\mathrm{BCTQ}$. The $\mathrm{DC}_{\text {caudate_R }}$ was found to be negatively correlated with the functional status scales $\left(\mathrm{R}^{2}=0.1627 ; P<0.05\right)$.

\section{Discussion}

The present study is the pioneered in investigating the topological properties of functional network in patients with CTS by using graph theory analysis. There were significantly different topologies between the CTS 

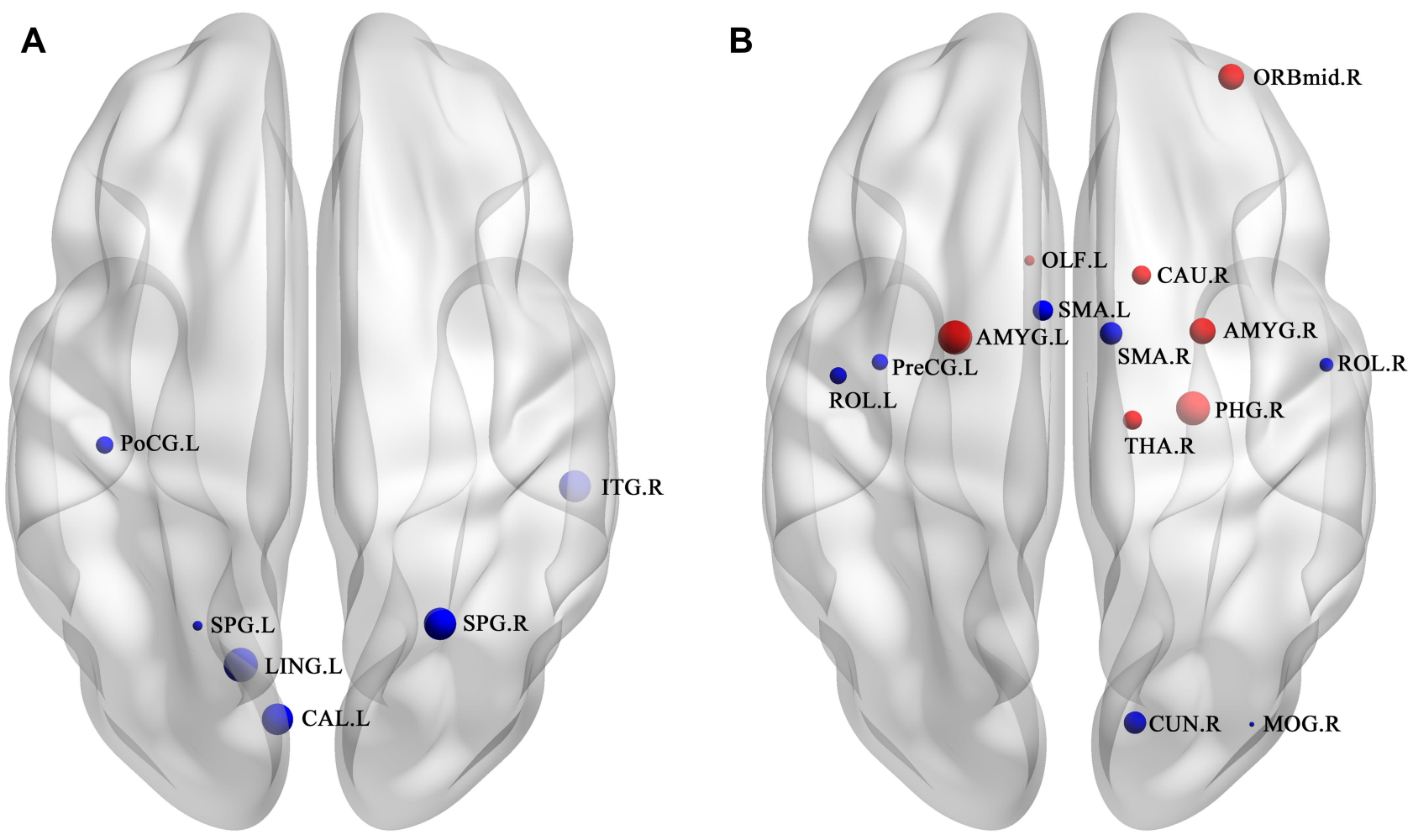

Figure 2 The differences in nodal clustering coefficient and degree centrality (DC) between carpal tunnel syndrome (CTS) patients and healthy control subjects (HCs). The statistical criterion for between-group differences was set at $p<0.05$, uncorrected. (A) Significantly altered nodal clustering coefficient; (B) significantly altered degree centrality. The red ball represent increased value of nodal properties while the blue balls represent decreased. The size of ball represent the significant difference.

Abbreviations: R, right; L, left; PoCG, postcentral gyrus; LING, lingual gyrus; SPG, superior parietal gyrus; PreCG, precentral gyrus; CAL, calcarine fissure and surrounding cortex; ITG, inferior temporal gyrus; ORBmid, middle frontal gyrus, orbital part; OLF, olfactory cortex; CAU, caudate nucleus; AMYG, amygdala; SMA, supplementary motor area; PHG, parahippocampal gyrus; THA, thalamus; ROL, rolandic operculum; CUN, cuneus; MOG, middle occipital gyrus.

patients and the HCs. Our major findings are as follows: (1) The networks of both CTS group and HCs contained small-worldness at a global level. Specifically, the CTS patients exhibited a decreased LP, suggesting the global integration of the functional brain network was chaotic and disturbed. (2) At the local level, significant alterations of several nodal properties were demonstrated between the CTS group and the HCs in basal gangliathalamo-cortical circuits and the pain matrix. These

Table 2 Brain Regions with Significant Nodal Shortest Path and Nodal Local Efficiency Differences Between the Two Groups

\begin{tabular}{|l|c|c|}
\hline Regional_Label & $\begin{array}{c}\text { Nodal Shortest } \\
\text { Path }\end{array}$ & $\begin{array}{c}\text { Nodal Local } \\
\text { Efficiency }\end{array}$ \\
\cline { 2 - 3 } & $\boldsymbol{P}$ & $\boldsymbol{P}$ \\
\hline Calcarine_L & 0.02105 & 0.01862 \\
Lingual_L & 0.02558 & 0.02842 \\
Postcentral_L & 0.0074 & 0.00459 \\
Parietal_Sup_L & 0.004 & 0.00791 \\
Parietal_Sup_R & 0.02243 & 0.04174 \\
Temporal_Inf_R & 0.02202 & 0.03191 \\
\hline
\end{tabular}

results provided new insights for improving our understanding of abnormal topological theory in the functional brain networks of CTS patients.

Table 3 Brain Areas with Significant Differences in Degree Centrality Between CTS and HCs

\begin{tabular}{|l|c|}
\hline & Degree Centrality \\
\hline Region Label & P \\
\hline Rolandic_Oper_L & 0.01489 \\
Rolandic_Oper_R & 0.02304 \\
ParaHippocampal_R & 0.00098 \\
Amygdala_L & 0.00095 \\
Amygdala_R & 0.00363 \\
Cuneus_R & 0.00660 \\
Occipital_Mid_R & 0.04058 \\
Precentral_L & 0.01658 \\
Frontal_Mid_Orb_R & 0.00378 \\
Supp_Motor_Area_L & 0.00889 \\
Supp_Motor_Area_R & 0.00639 \\
Olfactory_L & 0.03775 \\
Thalamus_R & 0.01094 \\
Caudate_R & 0.01081 \\
\hline
\end{tabular}




\section{Caudate_R}

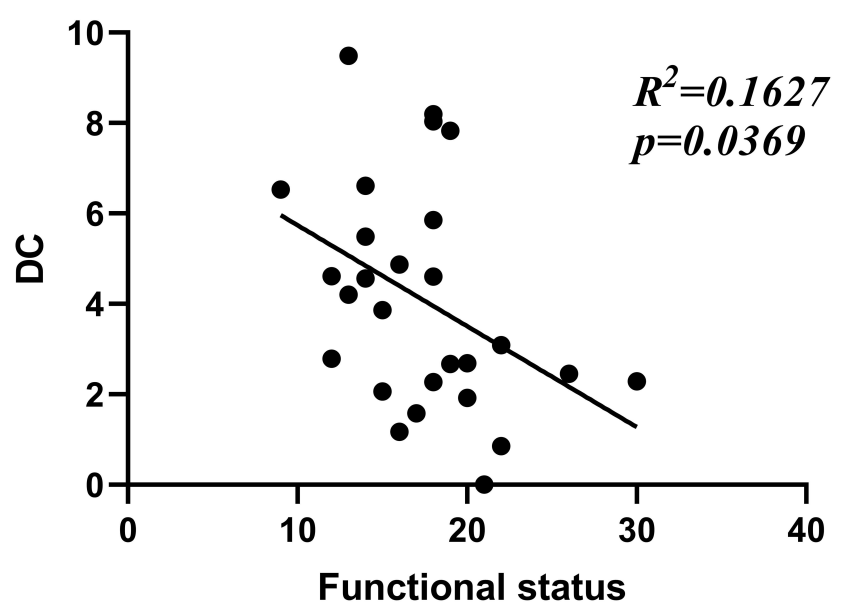

Figure 3 The correlation between the Boston symptom scores (daily life) of CTS patients and extent of degree centrality changes in the right caudate nucleus. Abbreviations: Caudate_R, right caudate nucleus; DC, degree centrality.

\section{Alterations at the Global Level}

The small-worldness existed in the functional networks of both CTS and HCs. Small-world networks are considered to be more efficient than regular and random networks, ${ }^{16}$ because they not only support specialized processing in local clusters, but also integrated processing over the entire network, and they even can maintain an optimal balance between the above two. Therefore, small-worldness is a meaningful characteristic for describing large-scale brain networks.

Our study showed that CTS patients displayed disrupted functional topological organization compared with that of HCs. The Lp and $\lambda$ of CTS group were significantly lower than those of HCs, the results may represent faster and more efficient information interactions between brain regions. Another prior PNI study also reported that the Lp was decreased in patients with brachial plexus injury. ${ }^{11}$ In addition, other chronic pain-related diseases showed a similar result, a shorter Lp, in the brain networks of patients. ${ }^{17}$ These relevant findings may indicate that pain reinforces the global connection and makes the networks more random. Our results may further identify and characterize the possible abnormal functional connectivity related to the global organization in the brains of CTS patients. These results may imply a stronger information processing capacity and more rapid information transfer at the global level in CTS patients compared with HCs.

\section{Alteration at the Local Level}

At the local level, we assessed these distant brain regions by different indexes of nodal properties (nodal clustering coefficient, degree centrality) and determined if the alteration of specific nodes leads to disorder in neural circuits. Nodal properties have been widely used to investigate multiple diseases, such as Alzheimer's disease, ${ }^{18}$ depression, ${ }^{19}$ and Parkinson's disease. ${ }^{20}$ The nodal clustering coefficient measures the connection strength between a node and its neighboring nodes. ${ }^{21,22} \mathrm{We}$ observed that the decreased nodal clustering coefficient in CTS patients was primarily in the left postcentral, calcarine, lingual cortex, and bilateral superior parietal cortex. Interestingly, all of these brain regions take part in the pain modulated processing.

Paresthesias, especially pain, is a major clinical feature of CTS. The postcentral gyrus, the primary sensory cortex (S1), majored in somatic sensory functions, particularly in relation to tactile discrimination and pain recognition. Previous studies have demonstrated that significant functional and structure alterations appear in the S1 of CTS patients, such as expanding the cortical representation of the digits $^{10}$ and a reduction in cortical thickness. ${ }^{6}$ Therefore, our findings supported the hypothesis that the connection between $\mathrm{S} 1$ and other regions was weakened.

Meanwhile, the superior parietal cortex plays a vital role in pain sensation, tactile discrimination, movement intention and cognitive regulation. ${ }^{23,24}$ Another study ${ }^{25}$ found that the bilateral superior parietal cortex shows significant deactivation in patients with chronic pain, and the authors speculated that this may be the reason for their enhanced cognitive and pain impairment. The inferior temporal gyrus is vital to the development and maintenance of chronic pain. ${ }^{26}$ Wang et al suggested that the gray matter volume of the inferior temporal is always decreased in patients with diverse pain syndromes compared with $\mathrm{HCs}^{27}$

Additionally, the calcarine gyrus as well as the lingual gyrus is involved in pain modulation and processing. ${ }^{28,29}$ Relevant studies have demonstrated that the decreased activation of the calcarine gyrus in patients with migraine is positively correlated with their visual analog scale scores. ${ }^{30}$ In the current study, the decreased nodal clustering coefficient in pain-related brain regions indicated that information transfer efficiency was obstructed between these regions and other areas within the network. We suspected that the reduced connection strength of the pain modulation-related regions resulted in the persistent pain.

Degree centrality describes the number of edges connecting to a node, ${ }^{31}$ and it represents the most local and directly quantifiable centrality measure. A higher DC represents more direct connections with the node. 
Interestingly, the anatomical structures with a changed DC constituted relatively complete neural pathways in this study. These significant regions mainly located in the frontal cortex, basal ganglia and thalamus. According to a previous study, ${ }^{32}$ the basal ganglia connects with thalamic and distinct frontal cortex regions, and the neural loops constituted by the connections between these regions were termed "basal ganglia-thalamo-cortical (BTC) circuits". To the best of our knowledge, PNI generally leads to the inactivation of the primary motor cortex and supplementary motor area. ${ }^{33,34}$

Motor areas are thought to be involved in the planning and execution of pain-induced scratching behavior. However, the thalamus and caudate are involved not only in motor mechanisms but also in pain-related action modulation. The thalamus plays a central subservient role in sensory information processing. ${ }^{35}$ It is a target region in the treatment of movement disorders and neuropathological pain. The caudate nucleus is a vital region associated with the neural mechanisms of goal-directed action. ${ }^{36}$ Tseng found that the thalamus was in a long-term activated condition in patients with trigeminal neuralgia. ${ }^{37}$ Stronger functional connectivity between the caudate and ipsilateral orbital frontal cortex was found in patients with pain. ${ }^{38}$ According to these theories, the increased DC in the thalamus and caudate could be caused by chronic pain. Interestingly, the result (Figure 3) of the correlation between $\mathrm{DC}_{\text {caudate } \mathrm{R}}$ value and $\mathrm{BCTQ}$ was negative. We speculated the dysfunction, such as motor, suppressed the activity the caudate which was excited by pain.

Pain also influences the emotional-affective regions that are involved in the pain matrix. ${ }^{39}$ The amygdala integrates pain signals to create affective memory, which promotes human learning to avoid harmful stimuli. ${ }^{40}$ Studies have suggested that persistent neuropathic pain may cause amygdala activation. ${ }^{41,42}$ Meanwhile, the middle orbital frontal gyrus also takes part in emotional memory. ${ }^{43}$ Otherwise, investigators have found that visual regions show reduced connectivity to other areas. ${ }^{44}$ The DCs in both the cuneus and the middle occipital gyrus were decreased, and this is supportive of the theory that pain causes visual changes. ${ }^{45}$ Additionally, one study also demonstrated that the parahippocampal is activated when pain is persistent and increased. ${ }^{46}$ In general, the changed DCs in the different brain regions represent the adaptive and integrated pain coping strategies in the sensory, motor, learning and vision neural circuits of the brain, and may be a manifestation of the increased global efficiency.
The limitations that should be addressed in this study. First, the confidence of these result may be reduced by the relatively small sample size. It is necessary to continue to recruit the participants in the following experiment. Second, the AAL, as one common anatomical template, was used to construct the brain functional network in this study. However, the brain region may be needed to parcellate finer and more complex to acquire a result in detail. Finally, only the BSTQ was used to assess the dysfunction of CTS patients, and although BSTQ is closely related to pain, a more detailed pain assessment questionnaire should be used to draw a more interesting relationship between pain and alterations of nodal properties in future investigations. ${ }^{47}$

In conclusion, the graph theoretic study of functional networks demonstrated that the CTS patients' brain with a higher global efficiency which represents stronger ability to rapidly combine specialized information from different brain circuits. And we observed significant alterations in these pain and motor processes-related brain regions that take part in making up BTC circuits and the pain matrix. The results provided effective complements to the neural mechanisms underlying CTS.

\section{Abbreviations}

CTS, carpal tunnel syndrome; rs-fMRI, resting-state functional magnetic resonance imaging; GRETNA, Graphtheoretical Network Analysis Toolkit; S1, the primary sensory cortex; PNI, peripheral nerve injury; HCs, healthy controls; DC, degree centrality; LP, characteristic path length.

\section{Informed Consent}

Written informed consent was obtained from each subject. This study was approved by the Medical. Ethics Committee of Yueyang hospital. All ethical work was performed in accordance with the Helsinki declaration. Informed consent was obtained from all individual participants included in the study.

\section{Acknowledgment}

This work was supported by the National Key R\&D Program of China [Grant No. 2018YFC2001600]; Shanghai Education Committee [Grant No. A2P1600325]; the Shanghai Rising-Star Program [Grant No. 19QA1409000]; Shanghai Municipal Commission of Health and Family Planning [Grant No.2018YQ02]; Shanghai "Rising Stars of Medical Talent" Youth Development Program [Grant No. RY411.19.01.10]. 


\section{Disclosure}

The authors declare that they have no competing interests.

\section{References}

1. Practice parameter for electrodiagnostic studies in carpal tunnel syndrome (summary statement). American Academy of Neurology, American Association of Electrodiagnostic Medicine, and American Academy of Physical Medicine and Rehabilitation. Neurology. 1993;43(11):2404-5. doi:10.1212/wnl.43.11.2404

2. Padua L, Padua R, Monaco ML, et al.; Italian CTS Study Group. Multiperspective assessment of carpal tunnel syndrome: a multicenter study. Neurology. 1999;53(8):1654. doi:10.1212/wnl.53.8.1654

3. Padua L, Coraci D, Erra C, et al. Carpal tunnel syndrome: clinical features, diagnosis, and management. Lancet Neurol. 2016;15 (12):1273-1284. doi:10.1016/S1474-4422(16)30231-9

4. Jablecki CK, Andary MT, So YT, et al.; AAEM Quality Assurance Committee. Literature review of the usefulness of nerve conduction studies and electromyography for the evaluation of patients with carpal tunnel syndrome. Muscle Nerve. 1993;16(12):1392-1414. doi:10.1002/mus. 880161220

5. Lang E, Claus D, Neundörfer B, et al. Parameters of thick and thin nerve-fiber functions as predictors of pain in carpal tunnel syndrome. Pain. 1995;60(3):295-302. doi:10.1016/0304-3959(94)00131-w

6. Maeda Y, Kettner N, Kim J, et al. Primary somatosensory/motor cortical thickness distinguishes paresthesia-dominant from pain-dominant carpal tunnel syndrome. Pain. 2016;157 (5):1085-1093. doi:10.1097/j.pain.0000000000000486

7. Fernández-de-las-peñas C, de la Llave-rincón AI, Fernández-Carnero J, et al. Bilateral widespread mechanical pain sensitivity in carpal tunnel syndrome: evidence of central processing in unilateral neuropathy. Brain. 2009;132(6):1472-1479. doi:10.1093/brain/awp050

8. Maeda Y, Kettner N, Holden J, et al. Functional deficits in carpal tunnel syndrome reflect reorganization of primary somatosensory cortex. Brain. 2014;137(6):1741-1752. doi:10.1093/brain/awu096

9. Maeda Y, Kettner N, Sheehan J, et al. Altered brain morphometry in carpal tunnel syndrome is associated with median nerve pathology. Neuroimage Clin. 2013;2:313-319. doi:10.1016/j.nicl.2013.02.001

10. Napadow V, Kettner N, Ryan A, et al. Somatosensory cortical plasticity in carpal tunnel syndrome - a cross-sectional fMRI evaluation. Neuroimage. 2006;31(2):520-530. doi:10.1016/j. neuroimage.2005.12.017

11. Wang W, Lu YC, Tang WJ, et al. Small-worldness of brain networks after brachial plexus injury: a resting-state functional magnetic resonance imaging study. Neural Regen Res. 2018;13(6):1061-1065. doi:10.4103/1673-5374.233450

12. Singer W. Neuronal synchrony: a versatile code for the definition of relations? Neuron. 1999;24(1):49-65.

13. Bullmore ET, Sporns O. Complex brain networks: graph theoretical analysis of structural and functional systems. Nat Rev Neurosci. 2009;10(3):186-198.

14. Aerts H, Fias W, Caeyenberghs K, et al. Brain networks under attack: robustness properties and the impact of lesions. Brain. 2016;139 (12):3063-3083.

15. Zalesky A, Fornito A, Bullmore ET. Network-based statistic: identifying differences in brain networks. Neuroimage. 2010;53 (4):1197-1207.

16. Watts DJ, Strogatz SH. Collective dynamics of 'small-world' networks. Nature. 1998;393(6684):440-442. doi:10.1038/30918

17. Li K, Liu L, Yin Q, et al. Abnormal rich club organization and impaired correlation between structural and functional connectivity in migraine sufferers. Brain Imaging Behav. 2017;11(2):526-540. doi:10.1007/s11682-016-9533-6
18. Matsuda H. MRI morphometry in Alzheimer's disease. Ageing Res Rev. 2016;30:17-24.

19. Gong Q, He YJ. Depression, neuroimaging and connectomics: a selective overview. Biol Psychiatry. 2015;77(3):223-235. doi:10.1016/j.biopsych.2014.08.009

20. Guan X, Guo T, Zeng Q, et al. Oscillation-specific nodal alterations in early to middle stages Parkinson's disease. Transl Neurodegender. 2019;8(1):36.

21. Cho EB, Han CE, Seo SW, et al. White matter network disruption and cognitive dysfunction in neuromyelitis optica spectrum disorder. Front Neurol. 2018;9:1104.

22. Rubinov M, Sporns O. Complex network measures of brain connectivity: uses and interpretations. NeuroImage. 2010;52(3):1059-1069. doi:10.1016/j.neuroimage.2009.10.003

23. Reed CL, Klatzky RL, Halgren E. What vs. where in touch: an fMRI study. Neuroimage. 2005;25(3):718-726.

24. Andersen RA, Cui H. Intention, action planning, and decision making in parietal-frontal circuits. Neuron. 2009;63(5):568-583.

25. Matsuo Y, Kurata J, Sekiguchi M, et al. Attenuation of cortical activity triggering descending pain inhibition in chronic low back pain patients: a functional magnetic resonance imaging study. J Anesth. 2017;31(4):523-530. doi:10.1007/s00540-017-2343-1

26. Obermann M, Rodriguez-Raecke R, Naegel S, et al. Gray matter volume reduction reflects chronic pain in trigeminal neuralgia. NeuroImage. 2013;74:352-358. doi:10.1016/j.neuroimage.201 3.02 .029

27. Wang Y, Cao D-Y, Remeniuk B, et al. Altered brain structure and function associated with sensory and affective components of classic trigeminal neuralgia. Pain. 2017;158(8):1561-1570. doi:10.1097/j. pain. 0000000000000951

28. Tedeschi G, Russo A, Conte F, et al. Increased interictal visual network connectivity in patients with migraine with aura. Cephalalgia. 2016;36(2):139-147. doi:10.1177/0333102415584360

29. Schwedt TJ, Chiang CC, Chong CD, et al. Functional MRI of migraine. Lancet Neurol. 2015;14(1):81-91.

30. Wei H, Zhou X, Chen YC, et al. Impaired intrinsic functional connectivity between the thalamus and visual cortex in migraine without aura. J Headache Pain. 2019;20(1):116.

31. Zuo X, Ehmke R, Mennes M, et al. Network centrality in the human functional connectome. Cerebral Cortex. 2012;22(8):1862-1875. doi:10.1093/cercor/bhr269

32. Herrero MT, Barcia C, Navarro JM. Functional anatomy of thalamus and basal ganglia. Childs Nerv Syst. 2002;18(8):386-404.

33. Qiu T, Chen L, Mao Y, et al. Sensorimotor cortical changes assessed with resting-state fMRI following total brachial plexus root avulsion. J Neurol Neurosurg Psychiatry. 2014;85(1):99-105. doi:10.1136/ jnnp-2013-304956

34. Bhat DI, Devi BI, Bharti K, et al. Cortical plasticity after brachial plexus injury and repair: a resting-state functional MRI study. Neurosurg Focus. 2017;42(3):E14.

35. Smythies J. The functional neuroanatomy of awareness: with a focus on the role of various anatomical systems in the control of intermodal attention. Conscious Cogn. 1997;6(4):455-481. doi:10.1006/ccog.19 97.0315

36. Grahn JA, Parkinson JA, Owen AM. The cognitive functions of the caudate nucleus. Prog Neurobiol. 2008;86(3):141-155. doi:10.1016/j. pneurobio.2008.09.004

37. Tseng W, Tsai M-L, Iwata K, et al. Long-term changes in trigeminal ganglionic and thalamic neuronal activities following inferior alveolar nerve transection in behaving rats. $J$ Neurosci. 2012;32 (45):16051-16063. doi:10.1523/JNEUROSCI.1828-12.2012

38. Song W, Cao Z, Lang C, et al. Disrupted functional connectivity of striatal sub-regions in Bell's palsy patients. Neuroimage Clin. 2017;14:122-129. doi:10.1016/j.nicl.2017.01.008 
39. Peirs C, Seal RP. Neural circuits for pain: recent advances and current views. Science. 2016;354(6312):578-584.

40. Arana FS, Parkinson JA, Hinton E, et al. Dissociable contributions of the human amygdala and orbitofrontal cortex to incentive motivation and goal selection. $J$ Neurosci. 2003;23(29):9632-9638.

41. Huang J, Gadotti VM, Chen L, et al. A neuronal circuit for activating descending modulation of neuropathic pain. Nat Neurosci. 2019;22 (10):1659-1668. doi:10.1038/s41593-019-0481-5

42. Corder G, Ahanonu B, Grewe BF, et al. An amygdalar neural ensemble that encodes the unpleasantness of pain. Science. 2019;363(6424):276-281. doi:10.1126/science.aap8586

43. Tan Y, Wu X, Chen J, et al. Structural and functional connectivity between the amygdala and orbital frontal cortex in burning mouth syndrome: an fMRI study. Front Psychol. 2019;10:1700.
44. Kong J, Loggia ML, Zyloney C, et al. Exploring the brain in pain: activations, deactivations and their relation. Pain. 2010;148 (2):257-267. doi:10.1016/j.pain.2009.11.008

45. Shen W, Tu Y, Gollub RL, et al. Visual network alterations in brain functional connectivity in chronic low back pain: a resting state functional connectivity and machine learning study. Neuroimage Clin. 2019;22:101775.

46. Long X, Huang W, Napadow V, et al. Sustained effects of acupuncture stimulation investigated with centrality mapping analysis. Front Human Neurosci. 2016;10:510. doi:10.3389/fnhum.2016.00510

47. Kuner R, Flor H. Structural plasticity and reorganisation in chronic pain. Nat Rev Neurosci. 2017;18(1):20-30.

\section{Publish your work in this journal}

The Journal of Pain Research is an international, peer reviewed, open access, online journal that welcomes laboratory and clinical findings in the fields of pain research and the prevention and management of pain. Original research, reviews, symposium reports, hypothesis formation and commentaries are all considered for publication. The manuscript management system is completely online and includes a very quick and fair peer-review system, which is all easy to use. Visit http:// www.dovepress.com/testimonials.php to read real quotes from published authors. 